\title{
Influence of laser parameters on segregation of Nb during selective laser melting of Inconel 718
}

\author{
*Liang Wang ${ }^{1,2}$, Ran Cui ${ }^{1}$, Bin-qiang Li', Xue Jia', Long-hui Yao' ${ }^{1}$, Yan-qing Su, ${ }^{1,2}$, Jing-jie Guo ${ }^{1,2}$, Tong Liü \\ 1. School of Materials Science and Engineering, Harbin Institute of Technology, Harbin 150001, China \\ 2. National Key Laboratory for Precision Hot Processing of Metals, Harbin Institute of Technology, Harbin 150001, China \\ 3. HIT-Chungu Joint Research Center for Additive Manufacturing Materials, Anhui Chungu 3D Printing Institute of Intelligent Equipment and \\ Industrial Technology, Wuhu 241200, Anhui, China
}

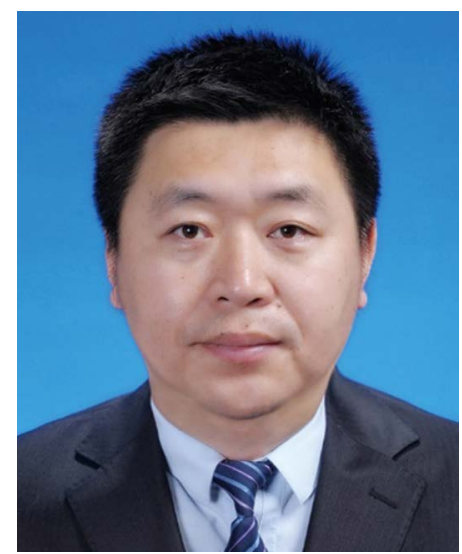

\section{*Liang Wang}

Ph. D., Professor. His research interests mainly focus on the solidification theory and technology of advanced materials. He has made achievements in the research of additive manufacturing process and liquid hydrogenation. He successfully prepared high strength $\mathrm{Al}$ alloy, NiTi shape memory alloy, and TiAl alloy, etc. by additive manufacturing. He found the hydrogen-induced purification phenomenon of titanium alloy, and revealed the mechanism of hydrogen-induced deoxidation of titanium alloy. He developed a new type of liquid hydrogenation equipment for preparation of titanium alloy with ultra-low oxygen content and to realize the recycle of titanium waste. $\mathrm{He}$ has participated in and completed more than 20 projects, including a 973 Project. He has been awarded the First Prize of the Technological Invention of Heilongjiang Province, and the Second Prize of the Natural Science of the Ministry of Education. To date, he has published $64 \mathrm{SCl}$ papers, and holds 8 authorized invention patents of China.

E-mail: wliang1227@hit.edu.cn

\begin{abstract}
A transient three-dimensional powder-scale model was established for understanding the flow field and mass transfer within the molten pool during the selective laser melting (SLM) of Inconel 718 alloy by considering some important physical phenomena, such as, a transition from powder to solid, nonlinearities produced by temperature-dependent materials' properties, and fluid flow in the calculation. The influence of laser power or scanning speed on the flow field and cooling rate was discussed in detail. The simulation results reveal that the motion of molten pool and higher cooling rate promote the mass transfer and benefit the solute distribution by increasing laser power. However, with increasing the scanning speed, the melt flow speed and cooling rate are elevated, resulting in an agglomeration of the solute elements, which is ascribed to the shorter dwelling time of liquid. Therefore, the segregation of $\mathrm{Nb}$ can be effectively suppressed by increasing laser power or decreasing scanning speed, which can decrease the dwelling time of liquid.
\end{abstract}

Key words: selective laser melting; Inconel 718 alloy; flow field; mass transfer; segregation

CLC numbers: TG146.1 5; Document code: A;

Article ID: 1672-6421(2021)04-379-10

\section{Introduction}

Additive manufacturing (AM) technologies enable the design optimization and structure complication for on-demand customized parts in the fields of aerospace, biomedical, and automotive industries ${ }^{[1,2]}$. Selective laser melting (SLM) is presently the most versatile AM process with advantages of high-precision and dense-solidification. The high power laser beam travels along the path that has been predesigned to selectively melt successive layers of fine metal powder deposited on the substrate and then the melted liquid quickly solidifies ${ }^{[3,4]}$. The whole process includes heat transfer, phase transformations, evaporation, fluid flow, mass transfer, and chemical reactions within the melt pool, which needs to be clarified using numerical simulation based on reliable physical models ${ }^{[5]}$. Many instructive results have been achieved, including the formation velocity of the molten pool which determined the existence of bubbles ${ }^{[6]}$, the optimization of the laser energy per unit length (LEPUL) which contributed to a flat melt pool surface ${ }^{[7]}$, and a proper molten pool size which helped to achieve a sound metallurgical bonding between the neighbor layers and tracks ${ }^{[8]}$.

Inconel 718 by SLM has attracted much attention for aerospace applications because of its high temperature yield strength, wear and rupture resistance, and corrosion and oxidation resistance ${ }^{[9,10]}$. Yi et al. ${ }^{[11]}$ reported both fine columnar and 
equiaxed grains appeared in Inconel 718 by SLM. In addition, it was also found the final microstructure and mechanical properties were sensitive to SLM parameters. The fracture surface observations indicated that the size of the dimples decreased and the number of dimples increased when the laser energy densities varied from 0.1 to $0.2 \mathrm{~J} \cdot \mathrm{mm}^{-1}{ }^{[12,13]}$. Wang et al. ${ }^{[14]}$ found the molten pool morphology had a considerable effect on microstructures. The greater the ratio of molten pool depth to width, the finer the grains. Jia et al. ${ }^{[15]}$ explored the microstructure evolution of SLM Inconel 718 alloy. It was found that with increasing the applied laser energy density, the morphology of the alloy changed from coarsened columnar to slender and uniformly columnar. Moreover, the high content of niobium $(\mathrm{Nb})$ in Inconel 718 could result in the segregation, causing macroscopic defects, such as freckles, which seriously deteriorate the solidified microstructure and the performance of the alloy ${ }^{[16,17]}$. Most studies on the SLM Inconel $718 \mathrm{did}$ not consider the segregation. The segregation is determined by the mass transport in the process of fluid flow and the cooling rate during rapid solidification ${ }^{[18,19]}$. Mei et al. ${ }^{[20]}$ studied the effect of welding speed on microsegregation in the process of electron-beam welded Inconel 718, and revealed that an insufficient solute transport and higher cooling rate occurred when the welding speed decreased, leading to an enhanced microsegregation. Long et al. ${ }^{[21]}$ analyzed the segregation of $\mathrm{Nb}$ in laser cladding Inconel 718 superalloy and concluded that low laser energy and cladding speed were beneficial for the high cooling rate to suppress the segregation of $\mathrm{Nb}$. However, few concerns focused on the $\mathrm{Nb}$ segregation with changing of the liquid flow, mass transfer and cooling rate in the process of SLM for Inconel 718.

In this study, the Inconel 718 was used as the starting powder particles, and a three-dimensional finite element simulation was carried out to investigate the effects of the laser power or laser scanning speed on the melt flow and mass transfer for the single-path of the single-layer during the SLM process.

\section{Model description}

To simplify the complicated issues during the SLM process, several assumptions are made in the model as follows: (1) The melt in the molten pool is laminar and incompressible homogeneous Newtonian fluid. (2) The free surface is flat, and the impact of the laser pressure is neglected. (3) The thermal physical parameters are temperature independent except the thermal conductivity, surface, viscosity, and specific heat.

(4) The evaporation of the melt pool is ignored.

\subsection{Physical model}

When an incident laser beam bears upon the powder surface, a tithe of energy is dissipated in the form of radiation and convection, while the majority of energy is absorbed by powder particles, as shown in Fig. 1. The absorbed energy melts the powder and renders a small-sized molten pool. The melted liquid rapidly solidifies at the cooling rate of $10^{3}-10^{8} \mathrm{~K} \cdot \mathrm{s}^{-1}$. Three coupling modes of heat transfer mechanism are

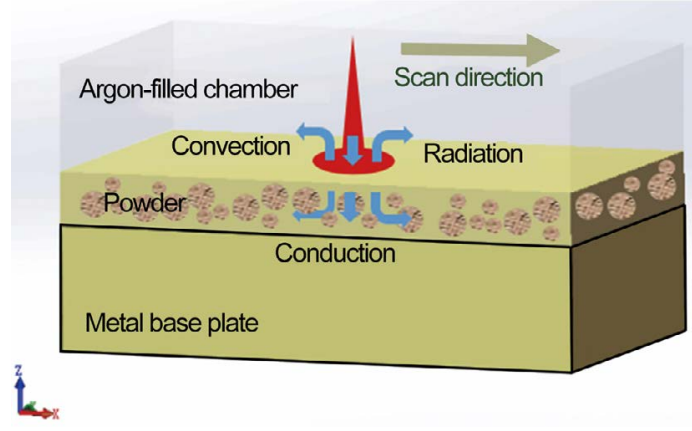

Fig. 1: Schematic of SLM physical model

considered during the process of SLM, which include heat radiation from laser beam to powder bed, heat conduction among powder particles, and heat convection between the powder bed and atmosphere. The complex physicochemical metallurgical phenomenon between the scanning laser beam and the powder bed is taken into account.

\subsection{Finite volumetric model (FVM)}

Figure 2 shows the FVM model of SLM process with a layer of Inconel 718 powder $(1.96 \mathrm{~mm} \times 0.98 \mathrm{~mm} \times 0.035 \mathrm{~mm})$ and 45 steel substrate $(1.96 \mathrm{~mm} \times 0.98 \mathrm{~mm} \times 0.14 \mathrm{~mm})$ under the argon protecting environment. The size of the argon model is $1.96 \mathrm{~mm}$ $\times 0.98 \mathrm{~mm} \times 0.04375 \mathrm{~mm}$. The refined gridding composed of 376,320 hexahedral cells, with the fine mesh of $0.00875 \mathrm{~mm}$ $\times 0.00875 \mathrm{~mm} \times 0.00875 \mathrm{~mm}$ is used in the whole model for capturing the gradients, such as speed, pressure, and temperature.

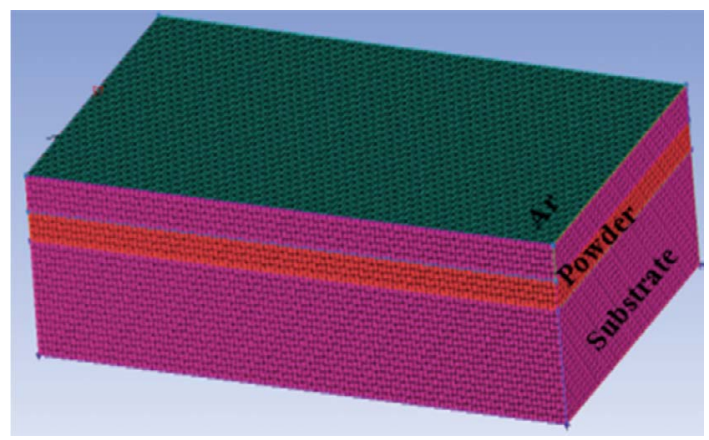

Fig. 2: Established three-dimensional mesh

\subsection{Governing equations}

The motion of the fluid generally follows the conservation laws of mass, momentum and energy, which can be indicated by the three-dimensional Cartesian coordinate as follows ${ }^{[22,23]}$.

(1) Mass conservation equation

$$
\frac{\partial \rho}{\partial t}+\nabla \cdot(\rho \vec{V})=M_{\mathrm{S}}
$$

where $\rho$ is the density of Inconel 718 alloy, $\vec{V}$ is the velocity of melt flow, and $M_{\mathrm{s}}$ is a mass source.

(2) Momentum conservation equation

$$
\rho\left(\frac{\partial \vec{V}}{\partial t}+\vec{V} \cdot \nabla \vec{V}\right)=\mu \nabla^{2} \vec{V}-\nabla P+M_{\mathrm{s}} \cdot \vec{V}+F
$$

where $\mu$ is the viscosity, $P$ is the pressure, and $F$ is the body force. 
(3) Energy conservation equation

$$
\begin{aligned}
& \rho\left(\frac{\partial \rho}{\partial t}+\vec{V} \cdot \nabla T\right)=\nabla \cdot(K \nabla T)+S_{\mathrm{H}} \\
& S_{\mathrm{H}}=-\left(\frac{\partial}{\partial t}(\rho \Delta H)+\nabla \cdot(\rho \vec{V} \Delta H)\right)
\end{aligned}
$$

where $k$ is thermal conductivity, $S_{\mathrm{H}}$ is the source term of the energy equation, and $\Delta H$ is the latent heat of phase transformation.

\subsection{Moving Gaussian heat source}

Gaussian volume heat source was chosen to characterize the SLM process. To obtain the movement of the heat source along the scanning direction, the equation of heat source $\left(q_{\text {laser }}\right)$ is written as:

$$
q_{\text {laser }}=\frac{3 P}{\pi R^{2} H} e^{\frac{-3\left(\left(x-\left(x_{0}+v t\right)\right)^{2}+\left(y-y_{0}\right)^{2}\right)}{R^{2}}}
$$

where $R$ is the radius of the Gaussian laser beam, $H$ is the action depth of the Gaussian laser beam, $P$ is the laser power, $x_{0}$ and $y_{0}$ are the initial position of the Gaussian laser beam, $t$ is the scanning time, and $v$ is the scanning speed.

\subsection{Initial and boundary conditions}

As soon as the laser beam travels along the predefined path at a constant velocity, many complicated thermal problems need to be solved. Proper initial and boundary conditions are expected to complete the accurate simulation.

In our model, the initial temperature is $300 \mathrm{~K}$ and the initial velocity of the melt flow is 0 at $t=0$.

The boundary condition on the free surface can be described as follows ${ }^{[24]}$ :

$$
-k \frac{\partial T}{\partial z}=q_{\text {laser }}-q_{\text {conv }}-q_{\text {radi }}
$$

where $q_{\text {conv }}$ is heat convection, $k$ is thermal conductivity, and $q_{\text {radi }}$ is heat radiation, expressed as:

$$
\begin{gathered}
q_{\text {conv }}=h_{\mathrm{c}}\left(T-T_{\infty}\right) \\
q_{\text {radi }}=\sigma_{\mathrm{e}} \varepsilon\left(T^{4}-T_{\infty}^{4}\right)
\end{gathered}
$$

where $h_{\mathrm{c}}$ is the convection coefficient, $\varepsilon$ is emissivity, $T$ is the temperature of the melt, $T_{\infty}$ is the ambient temperature, and $\sigma_{\mathrm{e}}$ is the Stefan-Boltzmann constant $\left(5.67 \times 10^{-8} \mathrm{~W} \cdot \mathrm{m}^{-2} \cdot \mathrm{K}^{-4}\right)$.

Due to the interaction between Gaussian distribution laser heat source and powder bed, a large temperature gradient is formed. The temperature gradient will lead to a surface tension gradient, accordingly causing a Marangoni flow, which is regarded as the main driving force of melt flow. To simulate the Marangoni flow, the surface tension can be defined as ${ }^{[25,26]}$ :

$$
\begin{aligned}
& -\mu \frac{\partial u}{\partial z}=\frac{\partial \gamma}{\partial T} \frac{\partial T}{\partial x} \\
& -\mu \frac{\partial v}{\partial z}=\frac{\partial \gamma}{\partial T} \frac{\partial T}{\partial y}
\end{aligned}
$$

where $\gamma$ is the surface tension. The ambient temperature is set up on the lateral surfaces, and the heat loss is assumed to be the radiation transfer. The thermal conduction from the powder layer to the metal substrate is greater than that from the powder layer to the gas environment, so the heat loss at the interface between the powder layer and the substrate is faster.

\subsection{Physical properties}

During the process of SLM, the metal powder undergoes a "powderliquid-solid" transition with the transfer of energy from laser beam to powder bed, exhibiting a nonlinear characteristic. The effective thermal conductivity $\left(k_{\text {eff }}\right)$ of the loose powder bed plays a considerable role in determining the results of simulation. In the present simulation, the powder particles are supposed to be spherical and packed in regular forms, so the general value of the thermal conductivity can be calculated as ${ }^{[27]}$ :

$\frac{k_{\text {eff }}}{k_{\mathrm{f}}}=(1-\sqrt{1-\phi})\left(1+\frac{\phi k_{\mathrm{r}}}{k_{\mathrm{f}}}\right) \sqrt{1-\phi}\left(\frac{2}{1-\frac{k_{\mathrm{f}}}{k_{\mathrm{s}}}}\left(\frac{1}{1-\frac{k_{\mathrm{f}}}{k_{\mathrm{s}}}} \ln \left(\frac{k_{\mathrm{s}}}{k_{\mathrm{f}}}\right)-1\right)+\frac{k_{\mathrm{r}}}{k_{\mathrm{f}}}\right)$

where $\phi$ is the fractional porosity of the powder bed, $k_{\mathrm{f}}$ and $k_{\mathrm{s}}$ are the thermal conductivity of the melt surrounding the powder particles and the solid, respectively, $k_{\mathrm{r}}$ is the thermal conductivity of the powder bed because of the radiation among particles, which is described by

$$
k_{\mathrm{r}}=4 F \sigma_{e} T^{3} x_{\mathrm{r}}
$$

where $x_{\mathrm{r}}$ is the average diameter of the powder particles, $T$ is the temperature of the powder particles, and $F$ is a view factor approximately taken as $1 / 3$.

\section{Experimental procedures}

Spherical Inconel 718 powder with diameter $\sim 35 \mu \mathrm{m}$ was used. The SLM system mainly consists of a $\mathrm{Yb}$ :YGA fiber laser with a maximum power of $500 \mathrm{~W}$ and a spot size of $80 \mu \mathrm{m}$, an automatic metallic powder spreading device, an inert argon gas protection system, and a computer system for process control. The detailed process parameters are the same with those in the numerical simulation, as shown in Table 1. The samples were cut, ground, and polished followed by etching with a mixture of $\mathrm{H}_{2} \mathrm{O}, \mathrm{HCl}$, $\mathrm{HNO}_{3}$ and $\mathrm{H}_{2} \mathrm{O}_{2}$ at a ratio of $4: 4: 1: 2$. The composition analysis was conducted on a Quanta 200 FEG scanning electron microscope.

\section{Results and discussion}

\subsection{Characteristics of flow field}

As the laser beam travels along the single-scanning track, the distribution of temperature and velocity field tends to be in a stable condition. In this simulation, all the studies were carried out at the 100th time step (time step is $\sim 6 \times 10^{-6} \mathrm{~s}$ ). Figure 3 shows the computed transient temperature 
Table 1: Finite element simulation parameters

\begin{tabular}{cc} 
Parameters & Value \\
\hline The Stefan-Boltzmann constant, $\sigma\left(\mathrm{W} \cdot \mathrm{m}^{-2} \cdot \mathrm{K}^{-4}\right)$ & $5.67 \times 10^{-8}$ \\
Powder layer thickness, $d(\mu \mathrm{m})$ & 35 \\
Ambient temperature, $T_{0}(\mathrm{~K})$ & 293 \\
Laser power, $P(\mathrm{~W})$ & $110,130,150,170$ \\
Scan speed, $v\left(\mathrm{~m} \cdot \mathrm{s}^{-1}\right)$ & $1.15,1.45,1.75,2.05$
\end{tabular}

contours on the top view, longitudinal view, and crosssectional view of the melt pool with input laser power $(P)$ of $170 \mathrm{~W}$ at a scanning speed $(v)$ of $1.15 \mathrm{~m} \cdot \mathrm{s}^{-1}$. The area inside the liquidus of $1,635.14 \mathrm{~K}$ presents higher temperature, which induces a melt pool within this area. At $0.0006 \mathrm{~s}$, the laser beam stands at $1.005 \mathrm{~mm}$ along the $X$-axis. Melt pool and laser beam do not have the same center if observed from the top view. The center of the melt pool slightly offsets with $0.025 \mathrm{~mm}$ along the negative direction of the $X$-axis due to the thermal hysteresis when the laser beam leaves the certain (a)

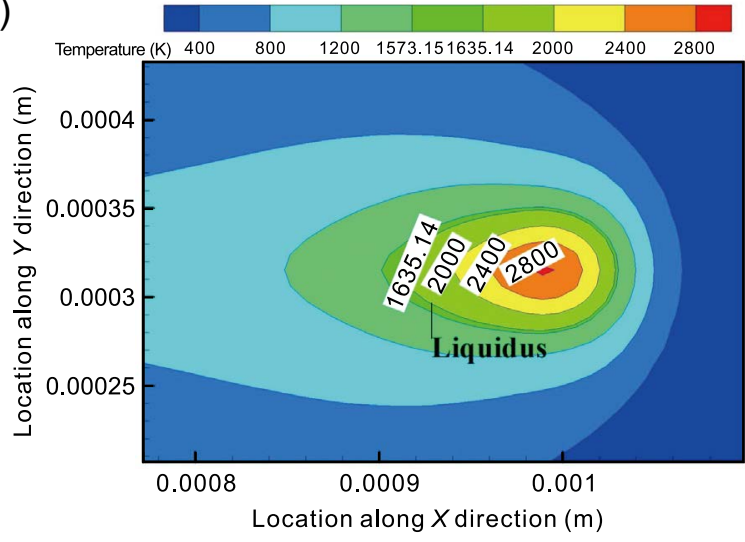

(b)

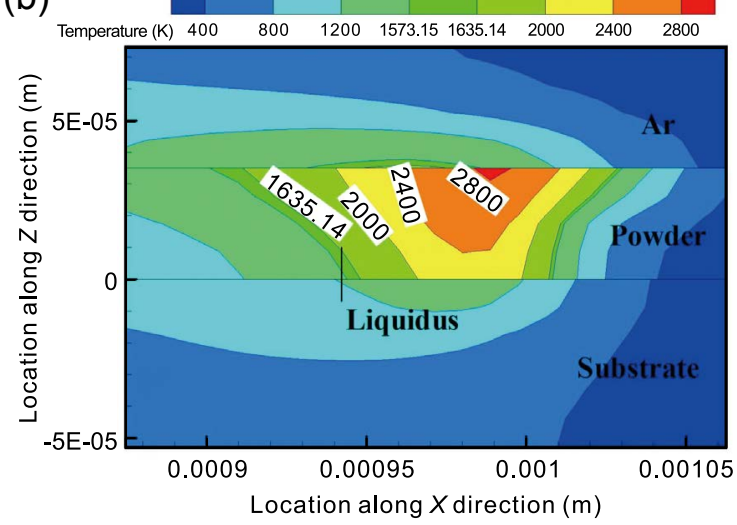

(c)

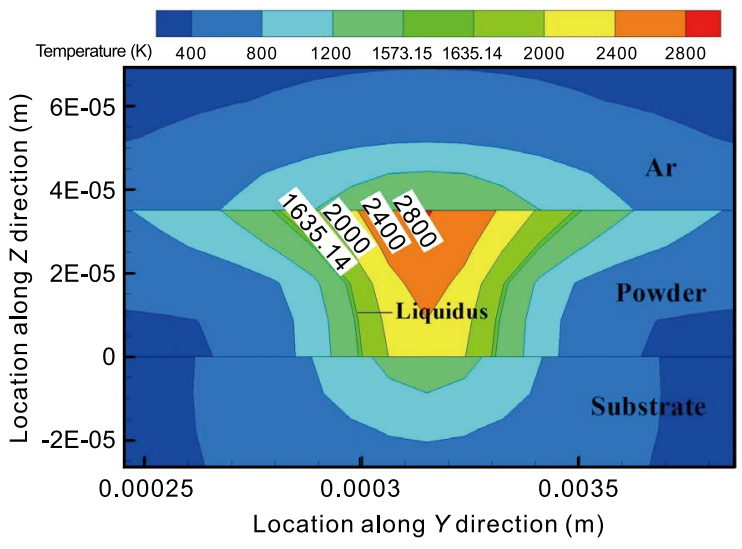

Fig. 3: Temperature plots in molten pool in different views: (a) top view; (b) longitudinal view; (c) cross-sectional view (laser power $170 \mathrm{~W}$, scan speed $1.15 \mathrm{~m} \cdot \mathrm{s}^{-1}$ )

position. The isotherm curves of the melt pool are similar to a series of ellipses affected by the moving of the heat source. The ellipses near the fore part are more intensive than those at the back end, resulting in a greater temperature gradient in the front of the melt pool. This can be attributed to the less heat convection in the liquid metal and less heat conduction between the fore part of melt pool and the solid powder particles because of the small front part of the melt pool. The contour plots are symmetrical in $X-Y, Y-Z$ sections, and the temperature value is maximum at the center of melt pool and decreases rapidly outward in all the three different sectional views due to the applied laser beam with Gaussian energy density distribution.

The distribution of temperature affects the flow field of the liquid. The velocity field in the three different views of the melt pool is presented in Fig. 4. Since the surface tension is in inverse proportion to the temperature, higher surface tension is available at lower temperature, leading to a surface tension gradient on the melt surface. As a result, Marangoni convection is induced when melt flows outward from the center to the edge of the melt pool under surface tension, as shown in Fig. 4(a). The convection flow is responsible for dissipating the thermal energy within the flowing liquid ${ }^{[28]}$. Assuming the fore part of the vector graph is a semi-circle, the radius $(R)$ represents the intensity of the Marangoni effect in the melt pool in terms of divergence of the velocity vector. A larger radius $(R)$ means a greater Marangoni effect. The melt flows upward in the center of the laser-induced melt pool owing to the combined action of gravity and buoyancy forces, as is depicted in Figs. 4(b) and (c). The vortexes are vividly presented in Figs. 4(b) and (c), indicating the existence of 

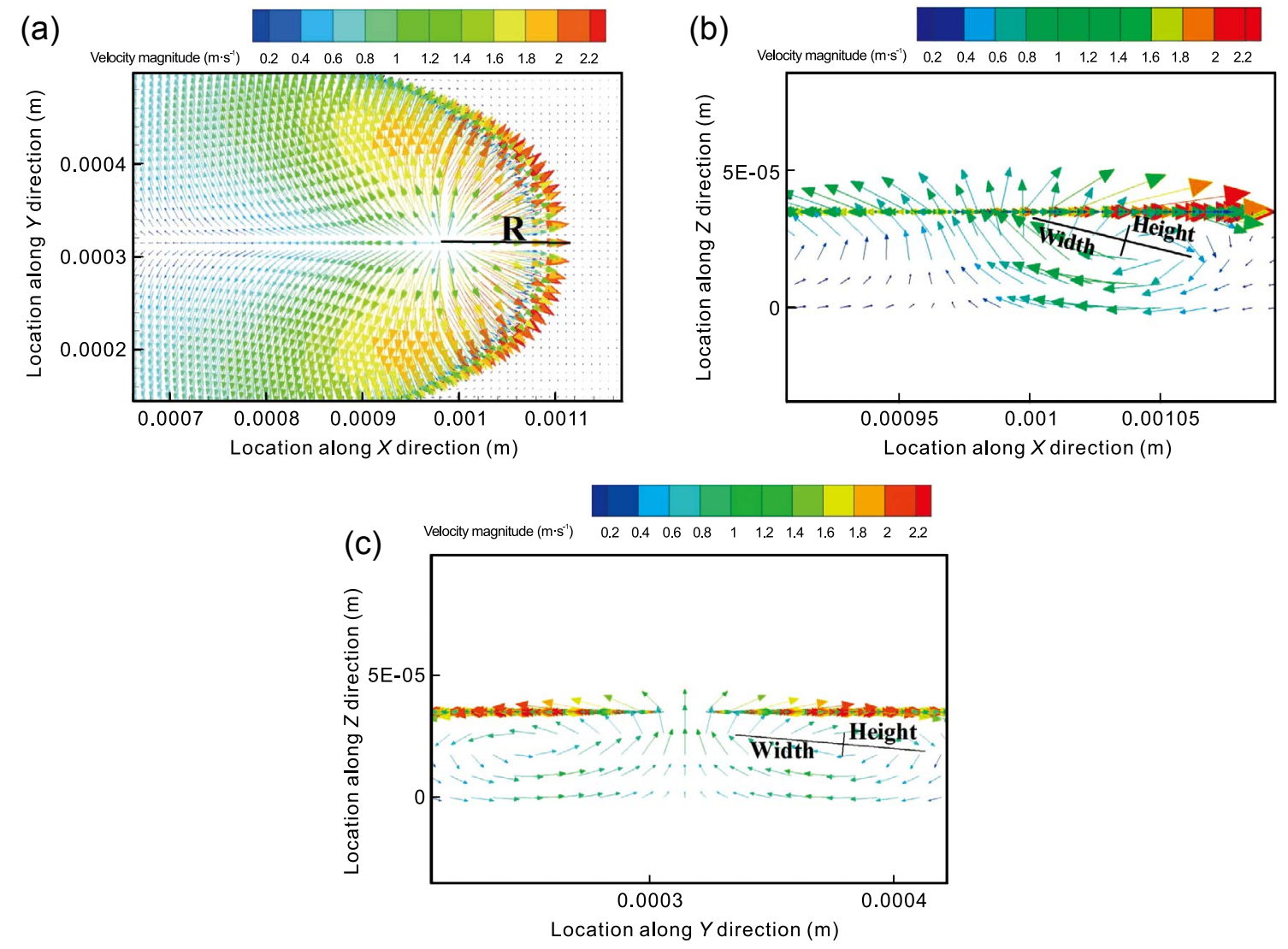

Fig. 4: Velocity vector plots in molten pool in different views: (a) top view; (b) longitudinal view; (c) cross-sectional view (laser power $170 \mathrm{~W}$, scan speed $1.15 \mathrm{~m} \cdot \mathrm{s}^{-1}$ )

heat convection in the melt pool. In the process of melt flows, the liquid suffers from an acting force that is exerted on by the front solid powder particles, gravity force and buoyancy force. With the combination of these forces, the formation of vortexes is inevitable. Moreover, the flow speeds are faster at the surface compared with those in the interior of the liquid metal because the motion of the melt pool originates at the surface due to the Marangoni convection ${ }^{[29]}$. The vortexes are similar with ellipses, so the width and height of the ellipse and the velocity magnitude can together represent how intensive the vortexes flow. The width is longer than the height in the formation of vortexes, suggesting the acting force from solid particles is the major driving force compared to the gravity and buoyancy forces.

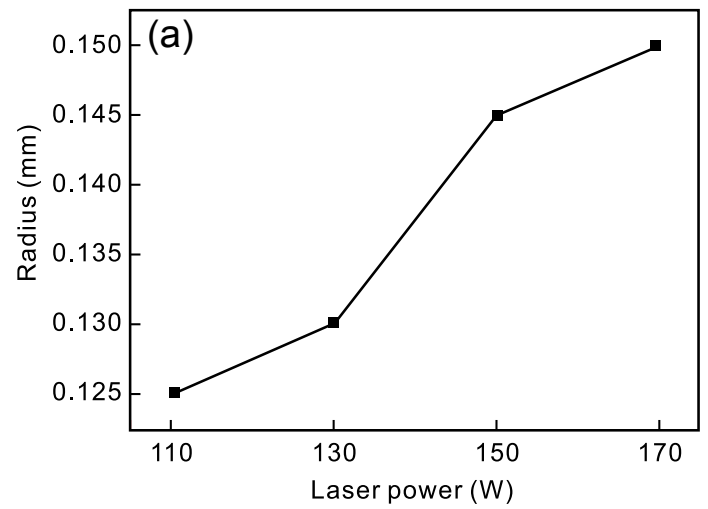

\subsection{Effect of laser parameters on flow field}

The radius $(R)$ of the vector graph on the top surface under different laser power is shown in Fig. 5(a). When the laser power increases from $110 \mathrm{~W}$ to $170 \mathrm{~W}$, radius increases from $0.125 \mathrm{~mm}$ to $0.15 \mathrm{~mm}$, which means a more intensive Marangoni effect at higher laser power. The velocity magnitude revolution profile of the melt flow is shown in Fig. 5(b). The speed curves of melt flow on the top surface along the $X$ direction are non-linear, indicating the complexity of the melt flow during SLM process. The maximum velocity magnitude elevates from $1.58 \mathrm{~m} \cdot \mathrm{s}^{-1}$ to $2.1 \mathrm{~m} \cdot \mathrm{s}^{-1}$ as the laser power increases from $110 \mathrm{~W}$ to $170 \mathrm{~W}$. The enhancement of the velocity magnitude further denotes

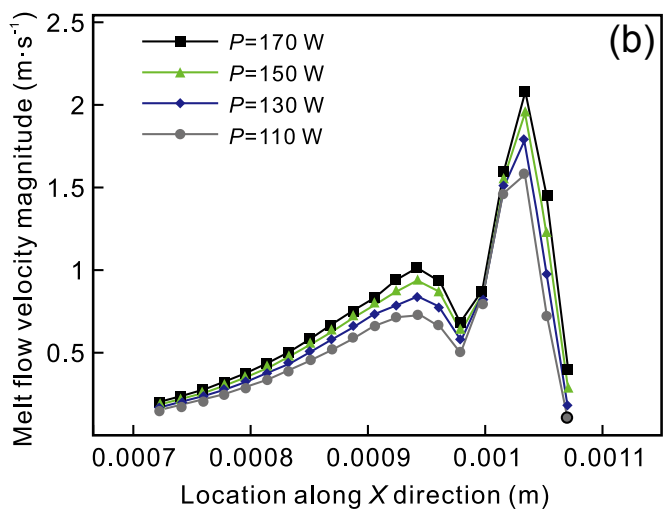

Fig. 5: Radius of vector graph (a) and velocity magnitude revolution profile of melt flow (b) on top surface under different laser power 
that the Marangoni effect indeed becomes more vigorous with increasing the laser power.

The characteristic of the melt flow is affected directly by the temperature distribution which is mainly dominated by the laser power. As the applied laser power enhances, both the operating temperature and temperature gradient increase gradually due to the heat accumulation in the powder bed. Accordingly, the dynamic viscosity decreases while the surface tension gradient increases. The combined effects of the lower dynamic viscosity and higher surface tension gradient promote the motion of the liquid. The mass transfer is mainly dominated by Marangoni convection, and the variation of the velocity magnitude reveals the migration of the mass transfer of the molten liquid materials ${ }^{[25,26]}$. Convective mass transfer alters solute concentration, leading to different morphologies of microstructures ${ }^{[27]}$. So, the higher laser power can facilitate the mass transfer in the melt pool during SLM process, which is beneficial for the solute distribution in the parts by SLM.

The simulated vortex dimensions dependent on laser power are

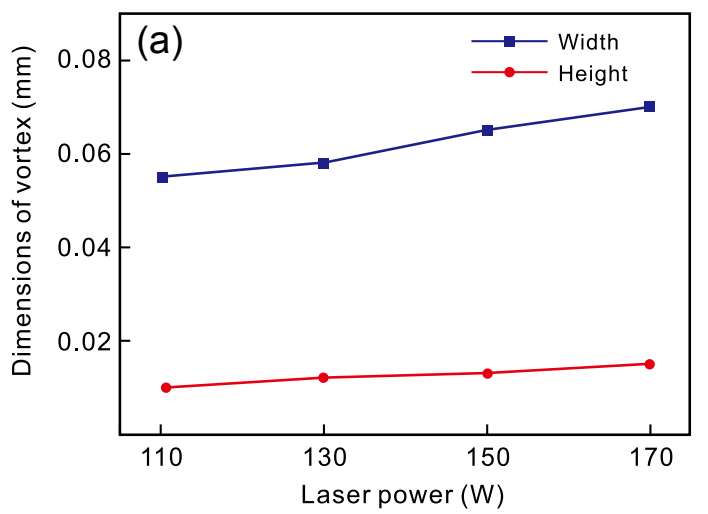

presented in Fig. 6(a). The increases in the width (from $0.055 \mathrm{~mm}$ to $0.07 \mathrm{~mm}$ ) and height (from $0.01 \mathrm{~mm}$ to $0.015 \mathrm{~mm}$ ) of the vortex are observed by increasing the laser power from $110 \mathrm{~W}$ to $170 \mathrm{~W}$. In addition, the calculated velocity magnitude of the vortex flow increases as the laser power elevates, which is depicted in Fig. 6(b). For a fixed scanning speed, an increase in laser power means the liquid moves forward more rapidly. Hence, the acting force coming from the front solid particles, which is the main driving force for the formation of the vortex, decreases at the edge of the melt pool. Consequently, the larger the vortex, the more intense the vortex flow generated. Mass transfer is dominated by convection when the liquid flows. The bigger size of the vortex and the more violent melt flows indicate the stronger Marangoni convection, contributing to the easier diffusion of the solute molecule. So, the higher laser power intensifies the mass transfer for the solute homogeneously distributed in the final parts.

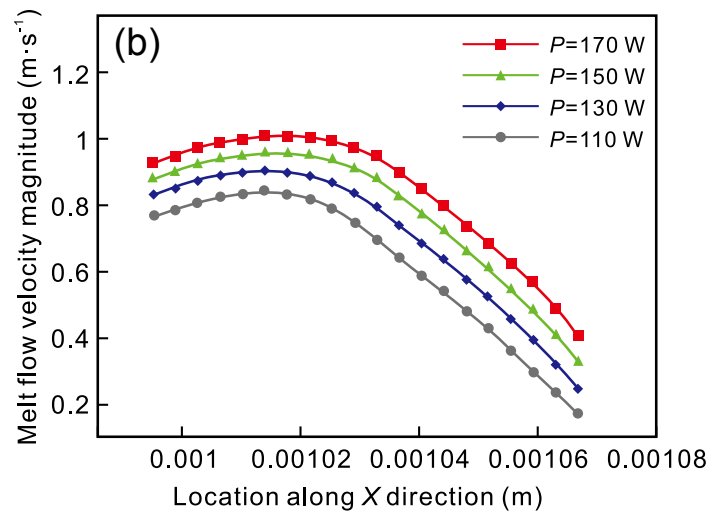

Fig. 6: Vortex dimensions (a) and maximum velocity magnitude of vortex flows (b) under different laser power

The radius $(\mathrm{R})$ of the vector graph on the top surface under different laser scanning speeds is shown in Fig. 7(a). The radius of the vector graph of $\sim 0.15 \mathrm{~mm}$ is obtained at a scanning speed of $\sim 1.15 \mathrm{~m} \cdot \mathrm{s}^{-1}$. When the scanning speed increases to $\sim 2.05 \mathrm{~m} \cdot \mathrm{s}^{-1}$, the generated radius of the vector graph decreases to $\sim 0.11$ $\mathrm{mm}$. Figure 7(b) depicts the variation of the velocity on the top surface under different scanning speeds. The maximum velocity decreases from $2.10 \mathrm{~m} \cdot \mathrm{s}^{-1}$ to $1.25 \mathrm{~m} \cdot \mathrm{s}^{-1}$ while the scanning

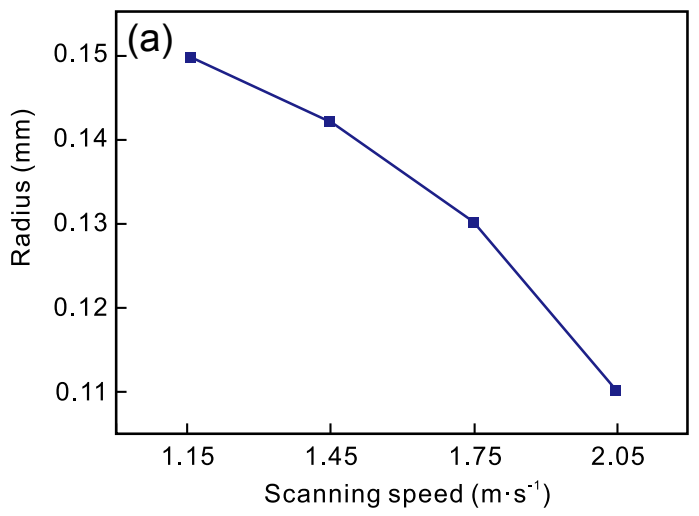

speed varies from $1.15 \mathrm{~m} \cdot \mathrm{s}^{-1}$ to $2.05 \mathrm{~m} \cdot \mathrm{s}^{-1}$. When the scanning speed increases, the interaction time between laser beam and powder bed decreases with the weakened heat accumulation in the powder bed, resulting in a decrement of the temperature and temperature gradient, which is responsible for the higher liquid dynamic viscosity and the lower surface tension gradient. Thus, mass transfer becomes non-significant on account of the increase of the laser scanning speed for a given laser power.

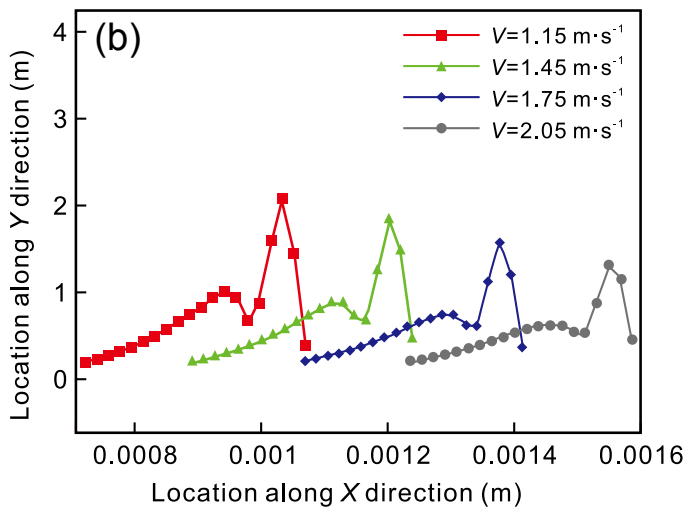

Fig. 7: Radius of vector graph (a), and velocity magnitude revolution profile of melt flow (b) on top surface under different scanning speeds 
The simulated vortex dimensions at different scanning speeds are shown in Fig. 8(a). The width (from $0.06 \mathrm{~mm}$ to $0.04 \mathrm{~mm}$ ) and height (from $0.02 \mathrm{~mm}$ to $0.016 \mathrm{~mm}$ ) decrease as the speed increases from $1.15 \mathrm{~m} \cdot \mathrm{s}^{-1}$ to $2.05 \mathrm{~m} \cdot \mathrm{s}^{-1}$. As depicted in Fig. 8(b), the velocities of the vortex flows are constrained as the scanning speed increases. Higher scanning

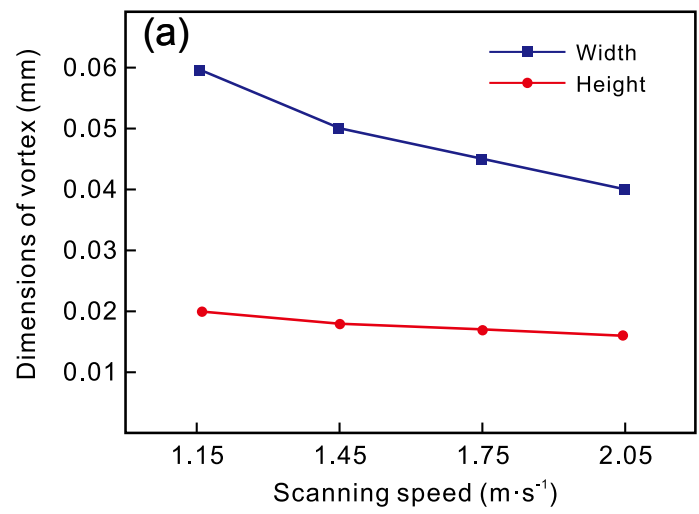

speed relaxes the melt flows because of the shortened acting force coming from front solid particles. The smaller size of the vortex and the relaxed melt flows restrain the mass transfer in the liquid, breaking the solute distribution in the final parts. Therefore, at the range of $1.15-2.05 \mathrm{~m} \cdot \mathrm{s}^{-1}$, a slower scanning speed benefits the mass transfer in the melt pool.

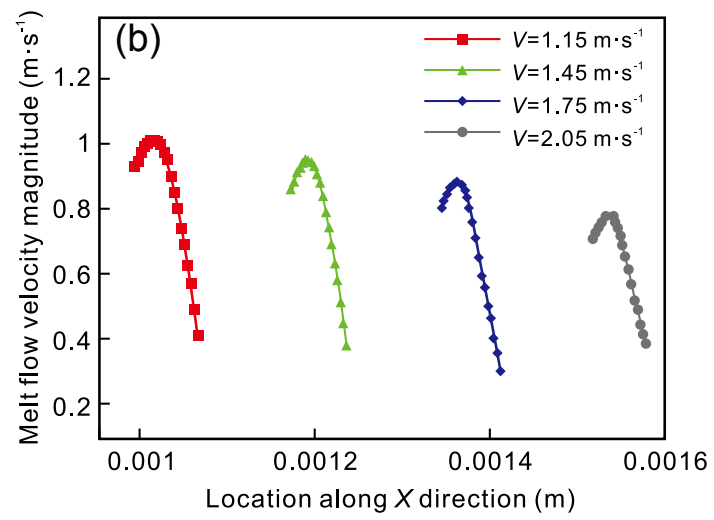

Fig. 8: Vortex dimensions (a) and maximum velocity magnitude of vortex flows (b) under different scanning speed

\subsection{Cooling rate and lifetime of liquid}

The time for the interaction between laser beam and powder bed has been proved to be extremely short in the process of SLM, leading to a higher heating/cooling rate (approx. $10^{6}-10^{7} \mathrm{~K} \cdot \mathrm{s}^{-1}$ ) and an ultrashort lifetime of liquid compared with a conventional process like die-casting. Laser power and laser scanning speed are considered effective to control the cooling rate and lifetime of liquid, as shown in Figs. 9 and 10. With increasing laser power (from $110 \mathrm{~W}$ to $170 \mathrm{~W}$ ), both the cooling rate (from $1.27 \times 10^{7} \mathrm{~K} \cdot \mathrm{s}^{-1}$ to $2.40 \times 10^{7} \mathrm{~K} \cdot \mathrm{s}^{-1}$ ) [Fig. 9(a)] and the lifetime of liquid (from $0.066 \mathrm{~ms}$ to $0.11 \mathrm{~ms}$ ) [Fig. 9 (b)] increase. Higher laser power induces higher energy input, which can provide a sufficient time to heat the powder before solidification, thus the dwelling time of the molten pool is increased. Further, the higher temperature gradient caused by the higher laser power is the reason for the increased cooling rate.

With increasing the scanning speed from $1.15 \mathrm{~m} \cdot \mathrm{s}^{-1}$ to $2.05 \mathrm{~m} \cdot \mathrm{s}^{-1}$, the cooling rate increases from $2.40 \times 10^{7} \mathrm{~K} \cdot \mathrm{s}^{-1}$ to $2.80 \times 10^{7} \mathrm{~K} \cdot \mathrm{s}^{-1}$ [Fig. 10(a)], while the lifetime of liquid decreases from $0.11 \mathrm{~ms}$ to $0.051 \mathrm{~ms}$ [Fig. 10(b)]. The increased cooling rate is due to the diminished interaction time between laser beam and powder bed at a higher scanning speed. A lesser heat accumulation in the power bed at a higher scanning speed leads to a shorter lifetime of liquid.

To be noted is that the solidification of SLM process proceeds under a super cooling rate so that the diffusion of solute element in the solid and liquid is restrained, and the equilibrium liquid-solid solute distribution is limited near the liquid-solid interface. Thus, the solute trapping happens during the solidification ${ }^{[28,29]}$. For a given scanning speed, the higher laser power produces a higher cooling rate and a longer lifetime of liquid. A higher cooling rate has a tendency to shorten the amount of residual liquid fraction, and a longer lifetime of liquid can provide a sufficient time to promote the redistribution of solute. Therefore, when the scanning speed is fixed, the higher cooling rate combined with the longer lifetime of liquid can effectively abate congregating of solute elements after solidification. Nevertheless, it is interesting to note that a higher cooling rate and a shorter lifetime of liquid can be obtained by increasing scanning speed with a constant laser power. This combination will lead to the formation of non-homogeneous distribution of solute elements because of insufficient rearrangement of the solute element.
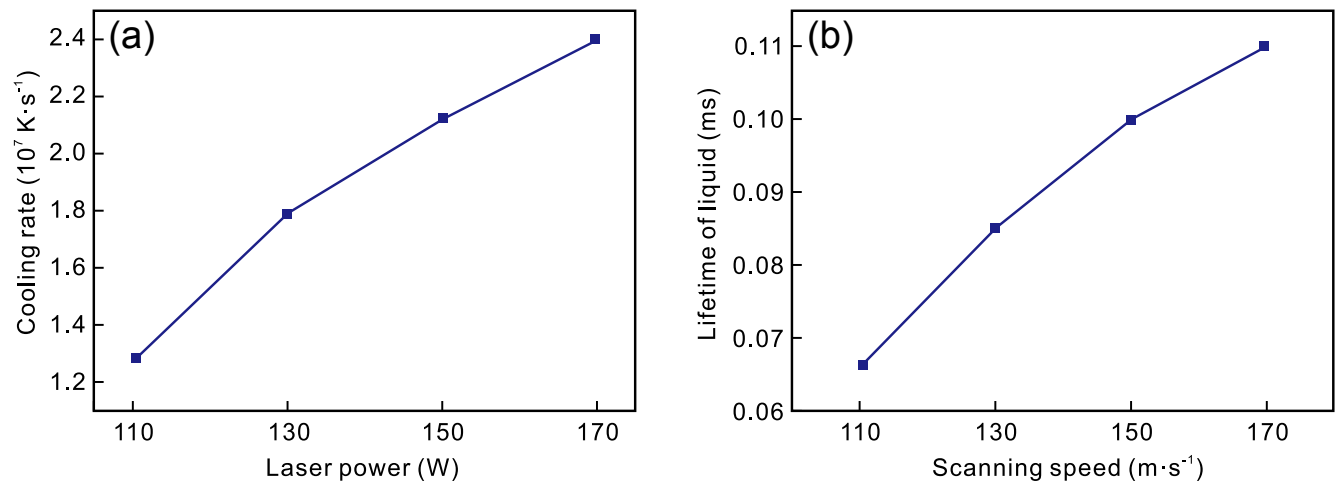

Fig. 9: Cooling rate (a) and lifetime of liquid (b) under different laser power 

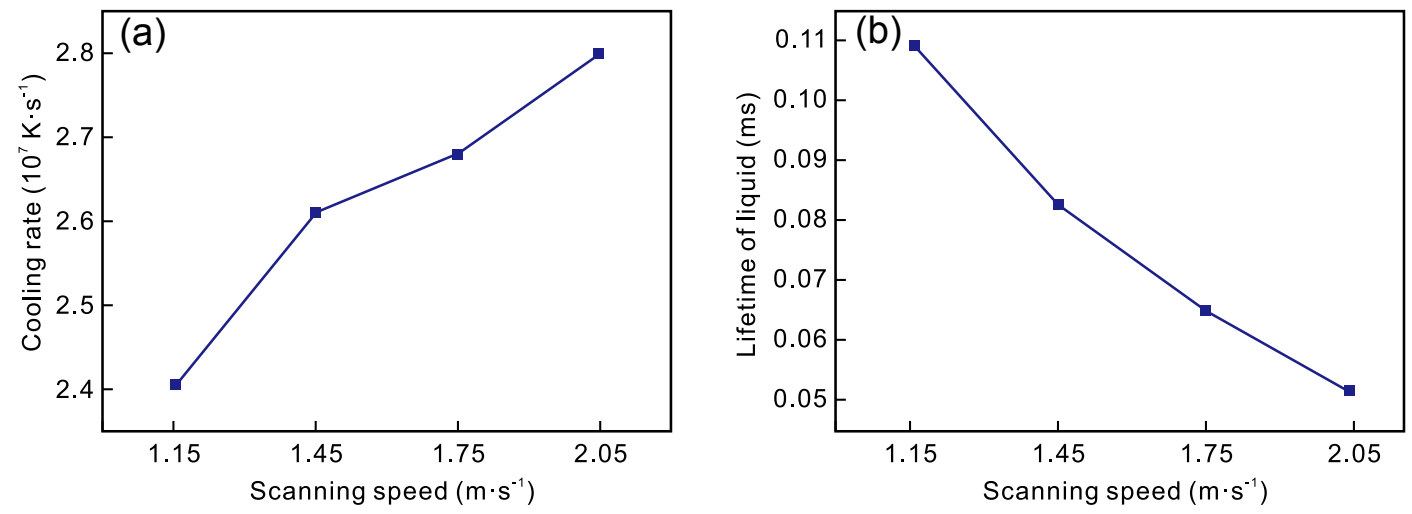

Fig. 10: Cooling rate (a) and lifetime of liquid (b) under different scanning speeds

\subsection{Experiment investigation}

SEM image for the cross-section of Inconel 718 after the melt pool solidifies is shown in Fig. 11(a). Positions 1 and 2 are selected to analyze the concentration of $\mathrm{Nb}$. Figure 11(b) presents the changed content of $\mathrm{Nb}$ with different laser power at a fixed scanning speed of $1.15 \mathrm{~m} \cdot \mathrm{s}^{-1}$ on the two positions. When the laser power is $110 \mathrm{~W}$, the content of $\mathrm{Nb}$ is $16.77 \%$ on Position 1 , and $10.5 \%$ on Position 2 . While the contents of $\mathrm{Nb}$ are close to each other when the laser beam increases to $170 \mathrm{~W}$, revealing that the distribution of $\mathrm{Nb}$ is more homogeneous with the increasing laser power. Figure 11(c) presents the changed content of $\mathrm{Nb}$ with the scanning speed at a fixed laser power of $170 \mathrm{~W}$ on the two positions. When the scanning speed is $1.15 \mathrm{~m} \cdot \mathrm{s}^{-1}$, the contents of $\mathrm{Nb}$ on the two positions are almost the same. However, when the scanning speed increases to $2.05 \mathrm{~m} \cdot \mathrm{s}^{-1}$, the content of $\mathrm{Nb}$ is $15.87 \%$ on Position 1 and $13.05 \%$ on Position 2, revealing that the distribution of $\mathrm{Nb}$ is more inhomogeneous with the increasing scanning speed.
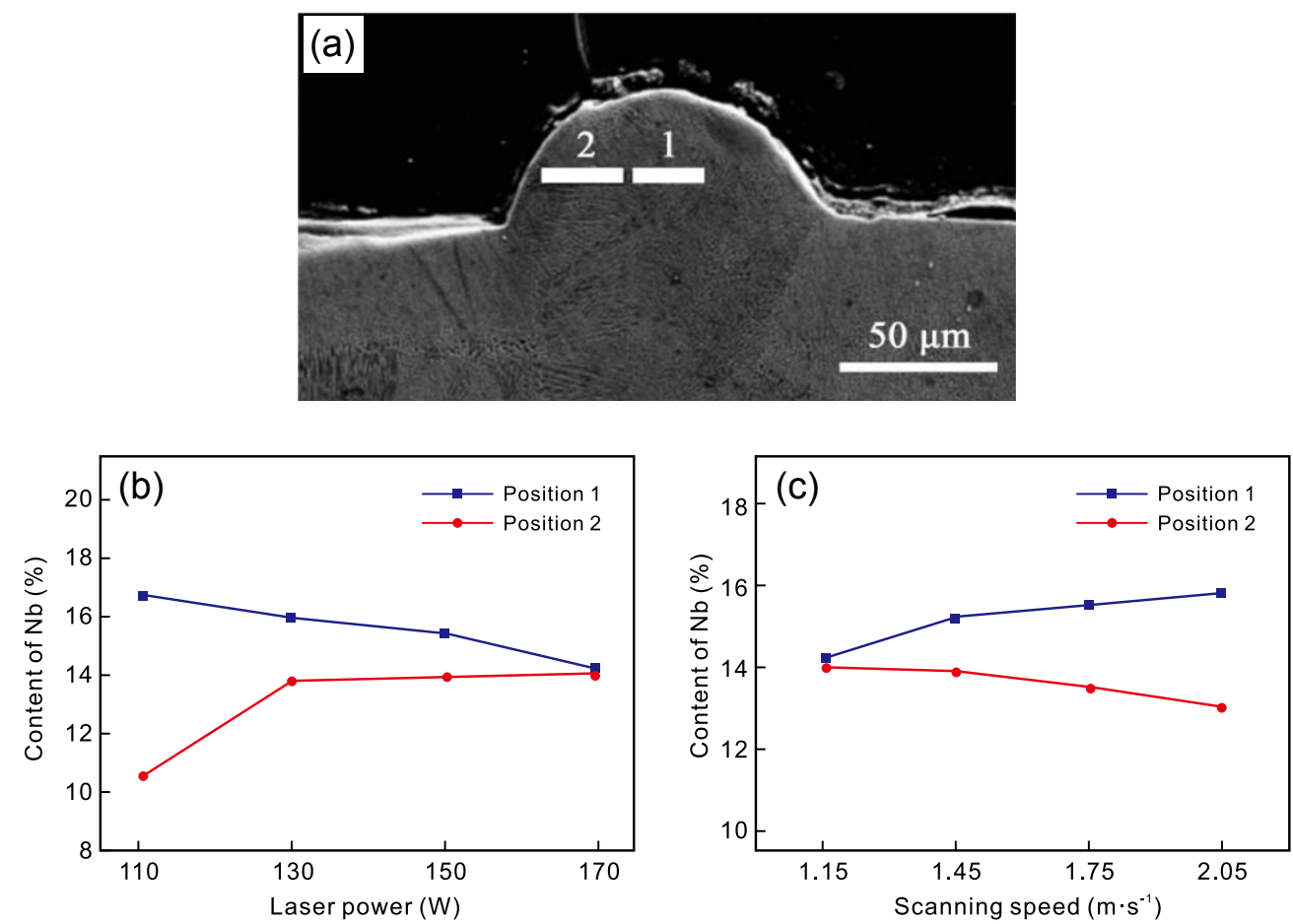

Fig. 11: SEM image for cross-section of Inconel 718 (a), content of $\mathrm{Nb}$ at Positions 1 and 2 under different laser powers (b) and scanning speeds (c)

During the rapid melting of SLM, a higher laser power or a slower scanning speed will cause a higher temperature gradient, and then a higher surface tension gradient promotes the melt flow resulting in the vigorous mass transfer, which is beneficial to the homogeneity of the elements. After melting, the melt pool begins to solidify with a superfast cooling rate. When the scanning speed is constant, the higher laser power can provide a higher cooling rate. On the one hand, the superfast cooling rate can induce the solute trapping to influence residual liquid fraction by affecting the duration time of solidification. With increasing the cooling rate, more solute trapping leads to less content of $\mathrm{Nb}$ in the residual liquid. On the other hand, the effective distributive coefficient of elements between solid and liquid phases tends to be closer 
to 1 with the increasing cooling rate. $\mathrm{Nb}$ is one of the positive segregation elements, so the fraction of solute decreases with the distributive coefficient increases. In addition, a higher laser power can spur a longer lifetime of liquid so that $\mathrm{Nb}$ can distribute in a sufficient time, contributing to the homogeneity of $\mathrm{Nb}$. However, when the laser power is fixed, although a higher cooling rate at a higher scanning speed can reduce the amount of residual liquid; the lifetime is so short that there is not enough time to rearrange $\mathrm{Nb}$, leading to a crosscurrent in the alloy.

\section{Conclusions}

The three-dimensional finite element model is established to mainly focus on the influences of flow field and cooling rate on the solute distribution by changing laser power or scanning speed, further revealing the segregation mechanism during SLM of Inconel 718. The results can be concluded as follows:

(1) The Marangoni convection is the main driving force to promote the melt flow within the melt pool. For a given scanning speed, a higher laser power enhances the melt flow rate and intensifies the mass transfer. For a given laser power, a higher scanning speed leads to a lower melt flow rate and relaxes the mass transfer.

(2) During the solidification, when the laser power increases at a fixed scanning speed, both the cooling rate and the lifetime of liquid increase, which contribute to a homogeneous distribution of solute. When the scanning speed increases at a fixed laser power, the cooling rate increases and the lifetime of liquid decreases, which result in a homogeneous distribution of solute.

(3) An experiment is designed to reveal the diffusion of $\mathrm{Nb}$ in Inconel 718 during the SLM process, showing a good agreement with the results predicted by simulation.

\section{Acknowledgements}

The authors gratefully appreciate the financial support from the National Natural Science Foundation of China (Nos. 51425402 and 51501048), and they acknowledge the significant help of Prof. Xing-jun Liu and Prof. Qian Zhang from Harbin Institute of Technology, Shenzhen.

\section{References}

[1] Huang $S \mathrm{H}$, Liu P, Mokasdar A, et al. Additive manufacturing and its societal impact: A literature review. The International Journal of Advanced Manufacturing Technology, 2013: 1-13.

[2] Kruth J P, Leu M C, Nakagawa T. Progress in additive manufacturing and rapid prototyping. CIRP AnnalsManufacturing Technology, 1998, 47: 525-540.

[3] Kruth J P, Froyen L, Van Vaerenbergh J, et al. Selective laser melting of iron-based powder. Journal of Materials Processing Technology, 2004, 149: 616-622.

[4] Su X, Yang Y. Research on track overlapping during selective laser melting of powders. Journal of Materials Processing Technology, 2012, 212: 2074-2079.
[5] Yuan P, Gu D. Molten pool behaviour and its physical mechanism during selective laser melting of TiC/AISi10Mg nanocomposites: Simulation and experiments. Journal of Physics D: Applied Physics, 2015, 48: 035303.

[6] Li C J, Tsai T W, Tseng C C. Numerical simulation for heat and mass transfer during selective laser melting of titanium alloys powder. Physics Procedia, 2016, 83: 1444-1449.

[7] Dai D, Gu D. Tailoring surface quality through mass and momentum transfer modeling using a volume of fluid method in selective laser melting of TiC/AISi10Mg powder. International Journal of Machine Tools and Manufacture, 2015, 88: 95-107.

[8] Shi Q, Gu D, Xia M, et al. Effects of laser processing parameters on thermal behavior and melting/solidification mechanism during selective laser melting of $\mathrm{TiC} / \mathrm{Inconel} 718$ composites. Optics \& Laser Technology, 2016, 84: 9-22.

[9] Sivaprasad K, Raman S G S. Influence of weld cooling rate on microstructure and mechanical properties of alloy 718 weldments. Metallurgical and Materials Transactions A, 2008, 39: 2115-2127.

[10] Knorovsky G, Cieslak M, Headley T, et al. Inconel 718: A solidification diagram. Metallurgical Transactions A, 1989, 20: 2149-2158.

[11] Yi J H, Kang J W, Wang T J, et al. Effect of laser energy density on the microstructure, mechanical properties, and deformation of Inconel 718 samples fabricated by selective laser melting. Journal of Alloys and Compounds, 2019, 786: 481-488.

[12] Holland S, Wang $X$, Chen J, et al. Multiscale characterization of microstructures and mechanical properties of Inconel 718 fabricated by selective laser melting. Journal of Alloys and Compounds, 2019, 784: 182-194.

[13] Wang L Y, Zhou Z J, Li C P, et al. Comparative investigation of small punch creep resistance of Inconel 718 fabricated by selective laser melting. Materials Science and Engineering: $A$, 2019, 745: 31-38.

[14] Wang H Y, Wang L, Cui R, et al. Differences in microstructure and nano-hardness of selective laser melted Inconel 718 single tracks under various melting modes of molten pool. Journal of Materials Research and Technology. 2020, 9: 10401-10410.

[15] Jia Q B, Gu D. Selective laser melting additive manufacturing of Inconel 718 superalloy parts: Densification, microstructure and properties. Journal of Alloys and Compounds, 2014, 585: 713721.

[16] Liu F, Lin X, Yang G, et al. Microstructure and residual stress of laser rapid formed Inconel 718 nickel-base superalloy. Optics \& Laser Technology, 2011, 43: 208-213.

[17] Liu W, Yao M, Chen Z, et al. Niobium segregation in Inconel 718. Journal of Materials Science, 1999, 34: 2583-2586.

[18] Wang Z, Guan K, Gao M, et al. The microstructure and mechanical properties of deposited-IN718 by selective laser melting. Journal of Alloys and Compounds, 2012, 513: 518-523.

[19] Chande T, Mazumder J. Two-dimensional, transient model for mass transport in laser surface alloying. Journal of Applied Physics, 1985, 57: 2226-2232.

[20] Mei Y, Liu Y, Liu C, et al. Effect of base metal and welding speed on fusion zone microstructure and HAZ hot-cracking of electron-beam welded Inconel 718. Materials \& Design, 2016, 89: 964-977.

[21] Long $Y$ T, Nie P L, Li Z G, et al. Segregation of niobium in laser cladding Inconel 718 superalloy. Transactions of Nonferrous Metals Society of China, 2016, 26: 431-436.

[22] Dai D, Gu D. Thermal behavior and densification mechanism during selective laser melting of copper matrix composites: simulation and experiments. Materials \& Design, 2014, 55: 482-491. 
[23] Raghavan N, Dehoff R, Pannala S, et al. Numerical modeling of heat-transfer and the influence of process parameters on tailoring the grain morphology of IN718 in electron beam additive manufacturing. Acta Materialia, 2016, 112: 303-314.

[24] Sarkar S, Raj P M, Chakraborty S, et al. Three-dimensional computational modeling of momentum, heat, and mass transfer in a laser surface alloying process. Numerical Heat Transfer: Part A: Applications, 2002, 42: 307-326.

[25] Rai R, Kelly S, Martukanitz R, et al. A convective heattransfer model for partial and full penetration keyhole mode laser welding of a structural steel. Metallurgical and Materials Transactions A, 2008, 39: 98-112.

[26] $\mathrm{Hu} \mathrm{Y,} \mathrm{He} \mathrm{X,} \mathrm{Yu} \mathrm{G,} \mathrm{et} \mathrm{al.} \mathrm{Heat} \mathrm{and} \mathrm{mass} \mathrm{transfer} \mathrm{in} \mathrm{laser}$ dissimilar welding of stainless steel and nickel. Applied Surface Science, 2012, 258: 5914-5922.

[27] Gu D, Yuan P. Thermal evolution behavior and fluid dynamics during laser additive manufacturing of Al-based nanocomposites: Underlying role of reinforcement weight fraction. Journal of Applied Physics, 2015, 118: 233109.

[28] Abderrazak K, Kriaa W, Salem W B, et al. Numerical and experimental studies of molten pool formation during an interaction of a pulse laser ( $\mathrm{Nd}$ : YAG) with a magnesium alloy. Optics \& Laser Technology, 2009, 41: 470-480.
[29] Manvatkar V, De A, DebRoy T. Heat transfer and material flow during laser assisted multi-layer additive manufacturing. Journal of Applied Physics, 2014, 116: 124905.

[30] Xia M, Gu D, Yu G, et al. Influence of hatch spacing on heat and mass transfer, thermodynamics and laser processability during additive manufacturing of Inconel 718 alloy. International Journal of Machine Tools and Manufacture, 2016, 109: 147157.

[31] Li Y, Gu D. Parametric analysis of thermal behavior during selective laser melting additive manufacturing of aluminum alloy powder. Materials \& Design, 2014, 63: 856-867.

[32] Steinbach $S$, Ratke L. The influence of fluid flow on the microstructure of directionally solidified AISi-base alloys. Metallurgical and Materials Transactions A, 2007, 38: 13881394.

[33] Wang L, Yao Y, Dong J, et al. Effect of cooling rates on segregation and density variation in the mushy zone during solidification of superalloy Inconel 718. Chemical Engineering Communications, 2010, 197: 1571-1585.

[34] Zhang Y, Li Z, Nie P, et al. Effect of cooling rate on the microstructure of laser-remelted Inconel 718 coating. Metallurgical and Materials Transactions A, 2013, 44: 55135521. 